\title{
Uso de morfina peridural em pacientes com disautonomia após traumatismo craniencefálico
}

\author{
Luis Roberto Mathias Junior', Wellingson Silva Paiva², Almir Ferreira de Andrade'3, \\ Robson Luis Oliveira Amorim ${ }^{4}$, Erich Talamoni Fonoff ${ }^{5}$, Manoel Jacobsen Teixeira ${ }^{6}$
}

Divisão de Neurocirurgia do Hospital das Clínicas da Faculdade de Medicina da Universidade de São Paulo (HCFMUSP)

\section{RESUMO}

Introdução: A disautonomia ou hiperatividade simpática paroxística (HSP) pode ocorrer em até um terço dos pacientes vítimas de trauma de crânio grave, entretanto ainda é uma entidade sem tratamento estabelecido. O uso de morfina intravenosa é descrito para o tratamento da HSP, sendo observada melhora principalmente na diminuição das frequências respiratória e cardíaca. Partindo desses preceitos, este estudo analisa os efeitos da morfina peridural nos pacientes com HSP. Pacientes e métodos: Foram avaliados três pacientes (dois masculino e um feminino), com idade média de 20,6 anos, vítimas de trauma de crânio grave, que apresentaram durante a evolução quadro clínico sugestivo de disautonomia. Os dados coletados incluíram detalhes do trauma, parâmetros fisiológicos, medicações utilizadas e evolução dos sintomas. Nesses pacientes, foi realizada infusão de morfina 4-6 mg/d no espaço peridural, em nível torácico, com média de 25,6 dias. Resultados: Dois pacientes tinham sinais radiológicos de lesão axonal difusa e um paciente tinha um volumoso hematoma extradural que foi operado. Os principais sintomas encontrados nos três pacientes foram sudorese, taquicardia, hipertermia e posturas distônicas. Em dois pacientes a hipertonia era global e em um paciente a postura distônica predominava no hemicorpo esquerdo. Após a passagem do cateter epidural com infusão de morfina, foi observada, já na primeira semana, melhora dos sintomas da hiperatividade simpática e da hipertonia nos grupos musculares envolvidos. Conclusão: $O$ uso de morfina peridural pode ser uma alternativa de tratamento nos pacientes com HSP refratários ao tratamento clínico.

\section{PALAVRAS-CHAVE}

Disautonomias, traumatismos encefálicos, morfina, lesão axonal difusa.

\section{ABSTRACT}

Use of peridural morphine in patients with posttraumatic autonomic disturbances

Introduction: The dysautonomia sympathetic hyperactivity or sympathetic storm syndrome (SSS) can occur in one third of patients with severe brain trauma, however, is still an entity without established treatment. The use of intravenous morphine is described for the treatment of SSS, and noted improvement mainly to lower frequencies of breathing and heartbeat. In this study we examine the effects of epidural morphine in patients with SSS. Patients and methods: We evaluated 3 patients (2 male, 1 female), with an average age of 20.6 years, victims of severe head trauma that presented SSS in hospital stay. The data collected included details of trauma, of physiology, medications used and symptoms. In these patients, infusion of morphine was realized 4 to $6 \mathrm{mg} / \mathrm{d}$ in the epidural space in the dorsal level with an average of 25.6 days. Results: Two patients had tomographic signs of diffuse axonal injury and one patient had a large acute epidural hematoma underwent to surgery. The main symptoms found in three patients, were dysautonomia, sweating, tachycardia, hyperthermia and postures distonics. In two patients, the stiffness was comprehensive and in one patient in the distonic posture in left side. After the procedure of the epidural catheter implant with infusion of morphine was found in the first week an improvement of symptoms of hyperactivity sympathetic. Conclusion: The use of epidural morphine can be an alternative treatment for sympathetic hyperactivity in patients with SSS.

\section{KEYWORDS}

Dysautonomia, craniocerebral trauma, diffuse axonal injury, morphine.

1. Médico-residente da Divisão de Neurocirurgia do HCFMUSP.

2. Médico-assistente e coordenador científico da Unidade de Emergência da Divisão de Neurocirurgia do HCFMUSP.

3. Professor livre-docente e coordenador da Unidade de Emergência da Divisão de Neurocirurgia do HCFMUSP.

4. Médico supervisor da Unidade de Emergência da Divisão de Neurocirurgia do HCFMUSP.

5. Coordenador do Grupo de Neurocirurgia Funcional.

6. Professor titular de Neurocirurgia da FMUSP e diretor da Divisão de Neurocirurgia do HCFMUSP. 


\section{Introdução}

A disautonomia ou hiperatividade simpática paroxística (HSP) pode ocorrer em até um terço dos pacientes vítimas de trauma de crânio grave, entretanto ainda é uma entidade sem tratamento estabelecido. ${ }^{1,2}$ É uma síndrome caracterizada por intensa agitação, diaforese, hipertermia, taquicardia e taquipneia, acompanhadas por hipertonia e postura extensora. ${ }^{1}$ Geralmente, essa síndrome manifesta-se precocemente, ainda nos cuidados de terapia intensiva, mas pode persistir durante a fase de reabilitação por semanas a meses. ${ }^{2} \mathrm{O}$ uso de morfina intravenosa é descrito para o tratamento da HSP, sendo observada melhora principalmente na diminuição das frequências respiratória e cardíaca. ${ }^{2,3}$ Partindo desses preceitos, este estudo analisa os efeitos da morfina peridural nos pacientes com HSP.

\section{Pacientes e métodos}

Três pacientes (dois masculino e um feminino), com idade média de 20,6 anos, vítimas de trauma de crânio grave, que apresentaram durante a evolução quadro clínico sugestivo de disautonomia, foram submetidos à implantação de cateter peridural para infusão de morfina na unidade de terapia intensiva (UTI) do pronto-socorro do Hospital das Clínicas da Faculdade de Medicina da Universidade de São Paulo. Todos os pacientes receberam tratamento clínico antes da infusão peridural. O implante foi realizado sob anestesia local, com pacientes posicionados em decúbito lateral. Uma incisão transversa era realizada no final das costelas torácicas e o subcutâneo era preparado para receber o reservatório. O cateter era inserido no espaço peridural por acesso percutâneo, na altura de T6. Os resultados eram monitorizados constantemente e, caso o paciente desenvolvesse qualquer reação adversa como vômitos ou retenção urinária, a concentração da droga era diminuída.

Os dados coletados incluíram detalhes do trauma, parâmetros fisiológicos, medicações utilizadas e evolução dos sintomas. Nesses pacientes, foi realizada infusão de morfina 2-8 mg/d no espaço peridural, em nível torácico, com média de 25,6 dias. das Clínicas da FMUSP, evoluindo com hematoma extradural temporoparietal à direita e necessidade de craniotomia descompressiva para drenagem do hematoma. No oitavo pós-operatório, apresentou sinais de disautonomia caracterizados por hipertensão, sudorese, taquicardia e espasticidade em hemicorpo à esquerda. No $15^{\circ}$ pós-operatório, foi passado cateter peridural para infusão de morfina (morfina $20 \mathrm{mg}+18 \mathrm{ml}$ de lidocaína $1 \%$, início de $1 \mathrm{ml}$ da solução de $12 / 12 \mathrm{~h}$, com aumento gradual). Melhora nos sinais de disautonomia (hipertensão, sudorese, taquicardia) já pode ser observada no segundo dia de infusão de morfina e importante controle das crises de hipertonia foi observado no nono dia de infusão. Permaneceu internada em UTI por 52 dias, com tempo para extubação definitiva de 37 dias, recebendo alta hospitalar consciente, contactuando com familiares.

\section{Caso 2}

Paciente do sexo masculino, 20 anos, vítima de acidente de moto versus ônibus. $\mathrm{Na}$ admissão em nosso pronto-socorro, encontrava-se anisocórico, E $>\mathrm{D}$, com postura de descerebração. Na tomografia de crânio, notavam-se inchaço cerebral associado à hemorragia subaracnoidea e lesão axonal difusa, e na tomografia cervical, evidenciava-se fratura de processo transverso (T10-L4). Optou-se por introdução de monitor de pressão intracraniana (PIC) e internação em UTI. A partir do oitavo pós-operatório, passou a apresentar sinais de disautonomia, caracterizados por hipertensão arterial, taquicardia sinusal, taquidispneia, febre, sudorese e espasticidade manifesta por hipertonia difusa. No $26^{\circ}$ pós-operatório, iniciou-se terapia para disautonomia e hipertonia com associação gradual de baclofeno, propranolol, diazepam e bromocriptina. No $36^{\circ}$ pós-operatório, foi instalado cateter peridural para infusão de morfina. Já na primeira semana de infusão, foi observado controle da frequência cardíaca e hipertermia e, após 18 dias de infusão, observou-se melhora considerável de todos os sinais de disautonomia, inclusive da hipertonia. Independência ventilatória foi verificada 27 dias após a instalação do cateter. Foi transferido posteriormente para outro serviço privado, dando sequência à reabilitação.

\section{Caso 1}

Paciente do sexo feminino, 15 anos, vítima de queda de bicicleta, admitido com 8 pontos na escala de coma de Glasgow (ECG), no pronto-socorro do Hospital

\section{Caso 3}

Paciente do sexo masculino, 22 anos, foi admitido com ECG 7 pontos, cuja tomografia de crânio mostrava 
sinais de lesão axonal difusa (LAD). Como conduta, optou-se por passagem de cateter para monitorização de pressão intracraniana, sendo em seguida admitido em UTI. Já na terceira semana de internação, evoluiu com sinais de disautonomia, caracterizados por sudorese intensa, hipertermia, distonia dos quatro membros. No $27^{\circ}$ pós-operatório, foi instalado cateter peridural para infusão de morfina, mantido por 18 dias. Como terapia complementar, foram utilizados propranolol, Haldol e baclofeno. Melhora significativa ocorreu no padrão distônico após o quinto dia de terapia peridural, permitindo desmame da ventilação não invasiva e extubação no oitavo dia de infusão peridural e redução marcante da terapêutica complementar. O paciente recebeu alta da UTI com escala de recuperação de Glasgow 3 em reabilitação neurológica.

\section{Resultados}

Os três pacientes deram entrada no PS com pontuação na ECG $<9$, caracterizando, portanto, alta gravidade, com necessidade de entubação e início de ventilação mecânica. Todos os três eram pacientes jovens, com idade inferior a 30 anos (Tabela 1).

Dois pacientes tinham sinais radiológicos de lesão axonal difusa e, como conduta inicial, iniciou-se monitorização de pressão intracraniana por meio de cateter intraventricular; um paciente tinha um volumoso hematoma extradural, que foi operado. Os principais sintomas disautonômicos encontrados nos três pacientes foram sudorese, taquicardia, hipertermia e posturas distônicas. Em dois pacientes, a hipertonia era global e em um paciente a postura distônica predominava no hemicorpo esquerdo. Para os três pacientes foi introduzida inicialmente terapêutica clínica, com pelo menos uma droga para o controle da espasticidade (baclofeno) e outra para o controle da disautonomia (propranolol), sem melhora clínica significativa. Outras medicações utilizadas no decorrer da terapia incluíram diazepam e bromocriptina. O tempo médio para a instalação do cateter peridural foi de aproximadamente 26 dias.

Após a passagem do cateter epidural com infusão de morfina, foi observada já na primeira semana uma melhora dos sintomas de hiperatividade simpática (sudorese, frequência cardíaca) e da hipertonia nos grupos musculares envolvidos. Nos três pacientes, foi possível independência ventilatória em menos de um mês após a instalação da morfina peridural. O tempo de permanência com o cateter variou de 18 a 62 dias, com média de 35,3 dias. Era infundida morfina a $2 \%$, nos três casos acompanhada de lidocaína de 1\%-2\%. O tempo de permanência em UTI variou de 33 até mais de 133 dias; para um dos pacientes a saída da UTI foi possível oito dias após a instituição da terapia peridural. Verificou-se ainda a redução da necessidade de medicações associadas após uma semana de implante do cateter peridural. Não se observou nenhuma complicação referente ao procedimento de colocação do cateter ou de sua permanência.

\begin{tabular}{|c|c|c|c|}
\hline & Caso 1 & Caso 2 & Caso 3 \\
\hline Sexo/Idade & Feminino/15 anos & Masculino/20 anos & Masculino/22 anos \\
\hline Início terapia peridural & $15^{\circ} \mathrm{PO}$ & $36^{\circ} \mathrm{PO}$ & $27^{\circ} \mathrm{PO}$ \\
\hline Duração da terapia em UTI & 52 dias & 133 dias & 35 dias \\
\hline Tempo para independência ventilatória & 37 dias & 83 dias & 33 dias \\
\hline Grupos musculares distônicos & Hemicorpo à esquerda & Hipertonia difusa & Hipertonia difusa \\
\hline Sintomas disautonômicos & Sudorese, hipertensão, taquicardia & $\begin{array}{l}\text { Hipertensão arterial, taquicardia } \\
\text { sinusal, taquidispneia, febre, sudorese }\end{array}$ & Sudorese, hipertermia, distonia \\
\hline ECG & 8 pontos & 4 pontos & 7 pontos \\
\hline Diagnóstico & HED TPD & Swelling + HSAt + LAD & LAD + hipóxia \\
\hline Cirurgia & Drenagem de hematoma & Monitor de PIC & Monitor de PIC \\
\hline Tempo de cateter & 26 dias & 62 dias & 18 dias \\
\hline Solução de morfina peridural & $\begin{array}{l}\text { Morfina } 2 \% 2 \mathrm{amp}+\text { lidocaína } \\
1 \% 18 \mathrm{ml}=>1 \mathrm{ml} 12 / 12 \mathrm{~h} \text { no } \\
\text { início com aumento gradual }\end{array}$ & $\begin{array}{l}\text { Lidocaína } 2 \% 12 \mathrm{ml}+\text { morfina } \\
2 \% 2 \text { amp }+6 \mathrm{ml} \mathrm{AD} \mathrm{=>} \mathrm{EV} 1 \mathrm{ml} \\
8 / 8 \mathrm{~h} \text { (com aumento gradual) }\end{array}$ & $\begin{array}{l}\text { Lidocaína } 2 \% 12 \mathrm{ml}+\text { morfina } \\
2 \% 2 \mathrm{amp}+6 \mathrm{ml} \mathrm{AD} \mathrm{=>} \mathrm{EV} 1 \mathrm{ml} \\
8 / 8 \mathrm{~h} \text { (com aumento gradual) }\end{array}$ \\
\hline Terapia complementar & $\begin{array}{l}\text { Baclofeno } 20 \mathrm{mg} / \mathrm{d} \\
\text { Diaxepam } 10 \mathrm{mg} / \mathrm{d} \\
\text { Propranolol } 40 \mathrm{mg} / \mathrm{d} \\
\text { Bromocriptina } 10 \mathrm{mg} / \mathrm{d}\end{array}$ & $\begin{array}{l}\text { Baclofeno } 20-70 \mathrm{mg} / \mathrm{d} \\
\text { Propranolol } 40 \mathrm{mg} / \mathrm{d} \\
\text { Diazepam } 5-30 \mathrm{mg} / \mathrm{d} \\
\text { Bromocriptina } 10 \mathrm{mg} / \mathrm{d}\end{array}$ & $\begin{array}{l}\text { Propranolol } 40 \mathrm{mg} / \mathrm{d} \\
\text { Haloperidol } 5 \mathrm{mg} / \mathrm{d} \\
\text { Baclofeno } 40 \mathrm{mg} / \mathrm{d} \\
\text { Diazepam } 20 \mathrm{mg} / \mathrm{d}\end{array}$ \\
\hline
\end{tabular}

PO: pós-operatório; ECG: escala de coma de Glasgow à admissão; HED: hematoma epidural; TPD: temporoparietal direita; HSAt: hemorragia subaracnoidea traumática; LAD: lesão axonal difusa; Fx: fratura; PIC: pressão intracraniana; AD: água destilada. 


\section{Discussão}

A fisiopatologia dessa síndrome disautonômica pode ser mais bem explicada por uma disfunção nos centros autonômicos dos diencéfalos ou suas conexões com regiões corticais, subcorticais e tronco cerebral. ${ }^{2}$ Ocorre, assim, um fenômeno de liberação pela perda dos centros de controle das funções vegetativas que incluem regulação da pressão arterial e temperatura. As drogas mais comumente usadas para o tratamento da disautonomia são morfina, bromocriptina, propranolol, clonidina, lorazepam e dantrolene. ${ }^{2,4}$ Rodriguez e cols. descrevem a aplicação de midazolan e baclofeno como agentes intravenosos preferenciais. ${ }^{4}$ A utilização de morfina peridural para espasticidade fornece bons resultados em pacientes com lesão medular, já que ela inibe os reflexos polissinápticos na medula espinal por meio de sua ação nos receptores opiáceos e induz analgesia e seu efeito sedativo é capaz de conter a taquicardia e a taquipneia. ${ }^{3} \mathrm{O}$ uso de morfina é controverso na literatura, contudo, tem se mostrado um instrumento para tratar pacientes com disautonomia severa quando aplicada por via endovenosa. Baguley e cols. ${ }^{5}$ descrevem, em estudo comparativo com 70 pacientes, o uso de morfina intravenosa com melhores resultados, contudo ressaltando os efeitos colaterais.

Tratamos três pacientes, vítimas de trauma de crânio grave, que apresentaram durante a evolução quadro clínico sugestivo de disautonomia, refratários à terapêutica clínica habitual. Os pacientes apresentaram considerável melhora do padrão de disautonomia, fato que aparentemente contribuiu para um desmame mais efetivo da ventilação mecânica, com redução do consumo energético.

\section{Conclusão}

O uso de morfina peridural pode ser uma alternativa de tratamento para os pacientes com HSP, principalmente para os que apresentam posturas extensoras, com benefício de menor proporção de efeitos colaterais. A elaboração de estudos controlados é importante para determinar o real papel da morfina peridural no tratamento desses pacientes.

\section{Referências}

1. Erickson DL, Blacklock JB, Michaelson M, Sperling KB, Lo JN. Control of spasticity by implantable continuous flow morphine pump. Neurosurgery. 1985;16:215-7.

2. Blackman JA, Patrick PD, Buck ML, Rust RS. Paroxysmal autonomic instability with dystonia after brain injury. Arch Neurol. 2004;61:321-8.

3. Rogano LA, Greve JM, Teixeira MJ. Use of intrathecal morphine infusion for spasticity. Arq Neuropsiquiatr. 2004;62(2-B):403-5.

4. Rodriguez N, Febrer A, Melenez M. Autonomic dysfunction in children with traumatic brain injury. Rev Neurol. 2006;43(3):143-6.

5. Baguley IJ, Cameron ID, Green AM, Slewa-Younan S, Marosszeky JE, Gurka JA. Pharmacological management of dysautonomia following traumatic brain injury. Brain Inj. 2004;18(5):409-17.

\footnotetext{
Endereço para correspondência:

Wellingson Silva Paiva

Rua Enéas Aguiar, 255, $4^{\circ}$ andar, Pinheiros

05403-010 - São Paulo

E-mail: wellingsonpaiva@yahoo.com.br
} 